Pacific Journal of Mathematics

WEAKLY ALMOST PERIODIC HOMEOMORPHISMS OF THE
TWO SPHERE

W. 


\title{
WEAKLY ALMOST PERIODIC HOMEOMORPHISMS OF THE TWO SPHERE
}

\author{
W. K. MASON
}

\begin{abstract}
A self-homeomorphism $f$ of the 2-sphere $S^{2}$ is weakly almost periodic (w.a.p.) if the collection of orbit closures forms a continuous decomposition of $S^{2}$. It is shown that if $f$ is orientation-preserving, w.a.p. and nonperiodic, then $f$ has exactly two fixed points, and every nondegenerate orbit closure is an homology 1-sphere. There is an example with an orbit closure which is an homology 1-sphere but not a real 1-sphere. If $f$ is orientation-reversing, w.a.p. and has a fixed point, then $f$ is shown to be periodic. The orbit structure of orientation-reversing, w.a.p., nonperiodic homeomorphisms on $S^{2}$ is studied.
\end{abstract}

1. Introduction. Let $f$ be a periodic mapping of the 2-sphere $S^{2}$ to itself. Kerékjártó [8] and Eilenberg [3] showed that $f$ is topologically equivalent either to the identity (every point fixed), to a rotation (two fixed points), a reflection (a simple closed curve of fixed points), or to a rotation followed by a reflection (no fixed points). If $f$ satisfies the weaker condition of being almost periodic (equivalent to having equicontinuous iterates), then the fixed point set of $f$ again is either empty or an $i$-sphere, $0 \leqq i \leqq 2$, [9]. (For related results on almost periodic mappings of subsets of $S^{2}$, see Hemmingsen [7].)

In the present paper we study the weakly almost periodic homeomorphisms on $S^{2}$, (the collection of orbit closures forms a continuous decomposition of $S^{2}$, and show that the set of fixed points is still either empty or an $i$-sphere, $0 \leqq i \leqq 2$, (Theorem 3 and Corollary 5). Some other results are: if $f: S^{2} \rightarrow S^{2}$ is weakly almost periodic (w.a.p.), orientation-reversing, and has a fixed point, then $f$ is periodic (Theorem 4); if $f: S^{2} \rightarrow S^{2}$ is w.a.p., orientation-preserving, and not periodic, then every nondegenerate orbit closure is an homology 1-sphere (Theorem 5).

A homeomorphism of $S^{2}$ to itself which is w.a.p. but not almost periodic is given in [12, Example 1]. This example is not almost periodic since it has an orbit closure which is not locally connected, (see [7, Section 5]). The collection of orbit closures, however, is easily seen to be continuous.

Our main theorems are given in $\S \S 6$ and 7 . Section 3 gives a summary of results in the theory of prime ends which we need. Section 4 discusses the fixed point theory used in $\S \S 5,6$, and 7 . (Those familiar with prime ends and local fixed point index may skip 
$\S \S 3$ and 4.) Many of our techniques are based on those of Cartwright and Littlewood in [2].

2. Definitions and notation. If $f: X \rightarrow X$ is a homeomorphism and $x \in X$, then the orbit closure of $x$ is the closure of the set of iterates $\left\{f^{n}(x)\right\}, n=0, \pm 1, \pm 2, \cdots,\left(f^{0}=I d\right)$.

The original definition of weakly almost periodic was given by Gottschalk in [5]. For compact spaces the original definition is equivalent to requiring that the orbit closures form a continuous decomposition [5, Theorem 5]. The equivalent definition which we shall use in our proofs is: $f: S^{2} \rightarrow S^{2}$ is weakly almost periodic if (a) the collection of orbit closures is a decomposition of $S^{2}$, (if two orbit closures meet, they are equal), and (b) for any closed set $B$, the union of all orbit closures which intersect $B$ is a closed set, [6, Theorem 4. 24, p. 34].

A point $x \in X$ is a nonwandering point if for every neighborhood $U$ of $x$, there is a nonzero integer $n$ such that $f^{n}(U) \cap U \neq \phi$. If $x$ is not a nonwandering point it is a wandering point. It is easily seen that if $f: S^{2} \rightarrow S^{2}$ is w.a.p. then every point is a nonwandering point.

A domain is a connected open set. If $A$ is a set $\mathrm{Cl}(A)$ and $\operatorname{Bd}(A)$ denote the closure and boundary, respectively, of $A$. If $U$ is a domain of $S^{2}$ and $x$ is a point in a component $R$ of $S^{2}-\mathrm{Cl}(U)$, then $\operatorname{Bd}(R)$ is the outer boundary of $U$ with respect to $x$.

An homology 1-sphere $K$ in $S^{2}$ is a continuum (closed, connected set) such that $S^{2}-K$ has exactly two components.

An open triod is a set homeomorphic to the set of all points $(x, y)$ in the plane such that either $-1<x<1$ and $y=0$, or $x=0$ and $0 \leqq y<1$. The points $(-1,0),(1,0),(0,1)$ are called the feet of the triod.

If $U$ is a domain then a crosscut of $U$ is an open arc in $U$ whose closure is an arc which intersects $\mathrm{Bd}(U)$ in two points. An endcut of $U$ is a half-open are in $U$ whose closure is an arc which intersects $\operatorname{Bd}(U)$ in one point.

3. Prime ends. In this section we state the results and definitions concerning prime ends which we shall use in $\S \S 5$ and 6 . The material in the present section is taken from [2], [11], and [15].

Let $U$ be a simply-connected domain in $S^{2}$ with a nondegenerate boundary. A $C$-transformation of $U$ onto the open unit disk $D$ is a homeomorphism $T: U \rightarrow D$ such that the image of any crosscut in $U$ is a crosscut in $D$, and the endpoints of such images of crosscuts 
of $U$ are dense in the boundary of $D$. The conformal mapping of $U$ onto $D$ given by the Riemann mapping theorem shows that $C$-transformations always exist. However, $C$-transformations may be constructed by topological methods, without using conformal mapping theory, [15, Appendix 2].

Given a homeomorphism $f$ of the closure of $U$ onto itself, and a $C$-transformation $T$ of $U$ onto $D$, we have that $T f T^{-1}: D \rightarrow D$ is a $C$-transformation which may be extended to a homeomorphism of the closed unit disk onto itself, $[15,(4.10)$ on page 6 , and (Al.7) on page 27].

A collection of crosscuts $Q_{1}, Q_{2}, \cdots$ of the simply connected domain $U$ is a chain if (a) the arcs $\operatorname{Cl}\left(Q_{1}\right), \mathrm{Cl}\left(Q_{2}\right), \ldots$ are pairwise disjoint, (b) $Q_{n}$ separates $Q_{n-1}$ from $Q_{n+1}$ in $U$, (c) there is a point on $\operatorname{Bd}(U)$ whose greatest distance from $Q_{n}$ approaches 0 as $n \rightarrow \infty$. Corresponding to each $Q_{n}$ there is a domain $G_{n}$ of $U-Q_{n}$ containing $Q_{n+1}$. Note that $G_{1} \supset G_{2} \supset \cdots$.

If $\left\{Q_{i}\right\},\left\{R_{i}\right\}$ are chains of crosscuts, and $\left\{G_{i}\right\},\left\{H_{i}\right\}$ are their respective corresponding domains, then $\left\{Q_{i}\right\},\left\{R_{i}\right\}$ are equivalent chains if for every $n$ there is an $m$ such that $H_{m} \subset G_{n}$ and $G_{m} \subset H_{n}$. Equivalent chains are said to define the same prime end. Thus, a prime end of $U$ is an equivalence class of chains of $U$.

If $Q_{1}, Q_{2}, \ldots$ is a chain of crosscuts in $U$, then their images $T\left(Q_{1}\right), T\left(Q_{2}\right), \cdots$ under the $C$-transformation $T: U \rightarrow D$ is a chain in $D$, [15, Appendix 2]. If $\left\{Q_{i}\right\}$ and $\left\{R_{i}\right\}$ are equivalent chains in $U$, then $\left\{T\left(Q_{i}\right)\right\}$, and $\left\{T\left(R_{i}\right)\right\}$ are equivalent chains in $D$, and in fact converge to the same point on the boundary of $D,\left(\left\{Q_{i}\right\}\right.$ and $\left\{R_{i}\right\}$ may not converge to the same point on $\operatorname{Bd}(U))$. Thus, $T$ sets up a $1-1$ correspondence between prime ends of $U$ and points of the unit circle $[11$, p. 621].

If $f: \mathrm{Cl}(U) \rightarrow \mathrm{Cl}(U)$ is a homeomorphism and $E$ is a prime end of $U$, then $E$ is fixed by $f$ if for some chain $\left\{Q_{i}\right\}$ defining $E$, we have that $\left\{Q_{i}\right\}$ and $\left\{f\left(Q_{i}\right)\right\}$ are equivalent chains. This definition is easily seen to be independent of which defining chain is used. If $T: U \rightarrow D$ is a $C$-transformation, $h: \mathrm{Cl}(D) \rightarrow \mathrm{Cl}(D)$ is the extension of $T f T^{-1}$, and $e$ is the point on $\operatorname{Bd}(D)$ corresponding to the fixed prime end $E$, then $h(e)=e$. Conversely, every fixed point of $h$ on $\operatorname{Bd}(D)$ corresponds to a fixed prime end of $f$.

If $E$ is a prime end of $U,\left\{Q_{i}\right\}$ is a defining chain for $E$, and $p$ is the point on $\operatorname{Bd}(U)$ to which the crosscuts $\left\{Q_{i}\right\}$ converge, then $p$ is a principal point of $E$. (We remark that there exists a $U$ with a prime end $E$ such that every point of $\operatorname{Bd}(U)$ is a principal point of $E$, [13].)

If $A$ is an endcut in $U$ with an endpoint $s \in \operatorname{Bd}(U)$, then there is a chain $\left\{Q_{i}\right\}$ defining a prime end $E$ such that $s$ is a principal 
point of $E$ and each crosscut $Q_{i}$ separates the endpoint of $A$ in $U$ from some (open) subarc of $A$ having $s$ as an endpoint. $E$ is the prime end determined by $A$. If $T: U \rightarrow D$ is a $C$-transformation, and $e$ is the point on $\operatorname{Bd}(D)$ corresponding to $E$, then $T(A)$ is an endeut in $D$ having $e$ as an endpoint, [15, page 5].

4. Lefschetz number and local fixed point index. In this section we state the results concerning fixed points which we shall use in $\S \S 5,6$, and 7 .

If $X$ is a compact polyhedron and $f: X \rightarrow X$ is a map (continuous function), then there is a certain rational number $L(f)$, called the Lefschetz number of $f$, associated with $f$ and $X,[14$, p. 195]. We shall use the following two facts about $L(f)$.

Fact 1. If $X$ is a two cell, then $L(f)=1$.

Fact 2. If $X$ is a 2-sphere and $f$ is an orientation-preserving homeomorphism, then $L(f)=2$.

For proofs of Facts 1 and 2, see [14, p. 196].

If $e$ is the category of compact polyhedra and maps, let $A(e)$ denote the set of pairs $(f, U)$, where $f: X \rightarrow X$ is a map in $e$ and $U$ is an open subset of $X$ such that $f$ has no fixed points on the boundary of $U$. Then there is a function $i$, the local fixed point index, from $A(e)$ into the rationals which satisfies the following axioms:

A1. If $(f, U),(g, U)$ belong to $A(e)$, and $f=g$ on the closure of $U$, then $i(f, U)=i(g, U)$.

A2. If $f_{t}$ is a homotopy such that $\left(f_{t}, U\right) \in A(e)$ for each $t$, $0 \leqq t \leqq 1$, then $i\left(f_{0}, U\right)=i\left(f_{1}, U\right)$.

A3. If $(f, U) \in A(e)$ and $U$ contains mutually disjoint open sets $V_{j}, j=1, \cdots, k$, such that $f$ has no fixed points on $U-\bigcup_{j=1}^{k} V_{j}$, then

$$
i(f, U)=\sum_{j=1}^{k} i\left(f, V_{j}\right) .
$$

In particular, if $f$ has no fixed points on $U, i(f, U)=0$.

A4. If $f: X \rightarrow X$ belongs to $e$, then $i(f, X)=L(f)$.

A5. If the maps $f: X \rightarrow Y, g: Y \rightarrow X$ belong to $e$, and

$$
(g f, U) \in A(e) \text {, }
$$


then $i(g f, U)=i\left(f g, g^{-1}(U)\right)$.

For further discussion of the local fixed point index see [4] or [1].

REMARK. If $D$ is the open unit disk, and $h$ is a map of the closure of $D$ to itself with no fixed points on $\operatorname{Bd}(D)$, then $i(h, D)=1$. For, by Fact 1 and Axiom A4, $1=\mathrm{L}(h)=i(h, \mathrm{Cl}(D))$. Then, by Axiom A3, $i(h, \mathrm{Cl}(D))=i(h, D)$.

5. Preliminary lemmas. Our first lemma is based on Lemma 11 of [2].

LemMa 1. Suppose $f: S^{2} \rightarrow S^{2}$ is a homeomorphism, $U$ is a simply connected domain with nondegenerate boundary, $f(U)=U$, and every point of $U$ is a nonwandering point. Suppose also that $E$ is a prime end of $U$ which is fixed by $f$. Then every principal point of $E$ is a fixed point of $f$.

Proof. Let $Q_{1}, Q_{2}, \cdots$ be a chain of crosscuts defining $E$ which converge to the principal point $p$ of $E$.

Case 1. $f\left(Q_{i}\right) \cap Q_{i}=\phi$ for some $i$. Let $V$ be the component of $U-Q_{i}$ containing $Q_{i+1}, Q_{i+2}, \cdots E$ is fixed by $f$, so $\left\{Q_{j}\right\}$ and $\left\{f\left(Q_{j}\right)\right\}$ are equivalent chains, hence $f(V) \cap V \neq \phi$. But then $f(V)$ either contains or is contained in $V$. Assume $f(V) \subset V$. Let $W$ be the nonempty open set $V-\mathrm{Cl}(f(V))$. Then $f^{n}(W) \cap W=\phi$ if $n \neq 0$. Thus no point of $W$ is a nonwandering point. This contradiction shows that Case 1 cannot occur.

Case 2. $f\left(Q_{i}\right) \cap Q_{i} \neq \phi$ for all $i, i=1,2, \ldots$. For each $i$, select a point $x_{i} \in Q_{i}$ such that $f\left(x_{i}\right) \in Q_{i}$. The crosscuts $Q_{1}, Q_{2}, \cdots$ converge to the principal point $p$, hence $\left\{x_{i}\right\} \rightarrow p$, hence $\left\{f\left(x_{i}\right)\right\} \rightarrow f(p)$. But $f\left(x_{i}\right) \in Q_{i}$, hence $\left\{f\left(x_{i}\right)\right\} \rightarrow p$. Hence $f(p)=p$ and the proof of Lemma 1 is complete.

Lemma 2. Suppose $f: S^{2} \rightarrow S^{2}$ is a homeomorphism, $M$ is an invariant continuum in $S^{2}$ which contains no fixed point of $f$, and every point of $S^{2}$ is a nonwandering point. Then $i(f, U)=1$ for every component $U$ of $S^{2}-M$ which is invariant under $f$. (See $\S 4$ for discussion of the fixed point index $i(f, U)$.)

Proof. Let $U$ be a component of $S^{2}-M$ such that $f(U)=U$. $M$ is connected, hence $U$ is simply connected. Also, $\mathrm{Bd}(U)$ is nondegenerate, since $M$ contains no fixed point of $f$. Let $T$ be a $C$ transformation of $U$ onto the open unit disk $D$. Extend $T f T^{-1}$ to a 
homeomorphism $h$ of $\mathrm{Cl}(D)$ onto itself. Since $\operatorname{Bd}(U)$ contains no fixed point of $f$, we see by Lemma 1 that $U$ has no fixed prime ends. Hence $h$ has no fixed points on $\operatorname{Bd}(D)$. Hence $i(h, D)=1$ by the Remark, $\S 4$.

We would like to conclude from Axiom A5 of $\S 4$ that $i(f, U)=1$. However, $D$ and $U$ are not compact polyhedra. We overcome this difficulty as follows: let $X$ be an open 2-cell which contains the fixed points of $f$ in $U$ and whose closure is contained in $U$. Let $Y$ be a closed 2-cell in $U$ containing $\mathrm{Cl}(X) \cup f(\mathrm{Cl}(X))$. Let $r_{1}: \mathrm{Cl}(D) \rightarrow T(Y)$, and $r_{2}: S^{2} \rightarrow Y$ be retractions. Since $T(X)$ contains all fixed points of $h$, we have:

$$
\begin{aligned}
1=i(h, D) & =i(h, T(X)) & & \text { by Axiom A3 } \\
& =i\left(T r_{2} f T^{-1} r_{1}, T(X)\right) & & \text { by } \mathrm{A} 1 \\
& =i\left(f T^{-1} r_{1} T r_{2}, X\right) & & \text { by A5 } \\
& =i(f, X) & & \text { by A1 } \\
& =i(f, U) & & \text { by A3. }
\end{aligned}
$$

The proof of Lemma 2 is complete.

6. Fixed point sets of weakly almost periodic homeomorphisms on $\mathrm{S}^{2}$.

THEOREM 3. Suppose $f: S^{2} \rightarrow S^{2}$ is a w.a.p. orientation-preserving homeomorphism. Then either $f$ is the identity or $f$ has exactly two fixed points.

Proof. Let Fix $(f)$ denote the set of fixed points of $f$. Assume $\operatorname{Fix}(f) \neq S^{2}$. Since $f$ is orientation-preserving it is easily seen that $f$ leaves every component of $S^{2}-\operatorname{Fix}(f)$ invariant, and so we may select an arc $A$ in one of these components such that $f(A) \cap A \neq \phi$. Denote by $M$ the union of all orbit closures which meet $A . M$ is closed, since $f$ is w.a.p.; $M$ contains no fixed point of $f$; and $M$ is connected since $M$ is the union of the connected set

$$
\bigcup_{n=-\infty}^{\infty} f^{n}(A)
$$

and limit points of this set.

Since $M$ and $\operatorname{Fix}(f)$ are disjoint closed sets, we see that $\operatorname{Fix}(f)$ is contained in a finite number $U_{1}, \cdots, U_{s}$ of components of $S^{2}-M$. By Axioms A3, A4, and Fact 2 of $\S 4$, we have

$$
2=L(f)=i\left(f, S^{2}\right)=\sum_{j=1}^{s} i\left(f, U_{j}\right) .
$$


But by Lemma $2, i\left(f, U_{j}\right)=1,1 \leqq j \leqq s$. Hence $s=2$.

It remains to show that $\operatorname{Fix}(f) \cap U_{j}, j=1,2$, is a single point.

Let $U$ be the component of $U_{1}-\operatorname{Fix}(f)$ with $\operatorname{Bd}\left(U_{1}\right) \subset \operatorname{Bd}(U)$. Since $\operatorname{Bd}\left(U_{1}\right)$ and $\operatorname{Fix}(f)$ are disjoint closed sets, we see that $\operatorname{Bd}(U)-$ $\operatorname{Bd}\left(U_{1}\right)$ is a closed nonempty subset of $\operatorname{Fix}(f)$.

Case 1. $\operatorname{Bd}(U)-\operatorname{Bd}\left(U_{1}\right)$ has more than one component. Then by [16, Corollary 3.11, p. 109], there is a simple closed curve $J$ in $U$ which separates $\operatorname{Bd}(U)-\operatorname{Bd}\left(U_{1}\right)$. Let $B$ be an arc with one endpoint on $\operatorname{Bd}\left(U_{1}\right)$, the other on $J$, and contained in $U$ except for one endpoint. Then $\operatorname{Bd}\left(U_{1}\right) \cup J \cup B$ is connected, and

$$
f\left(\operatorname{Bd}\left(U_{1}\right) \cup J \cup B\right) \cap\left(\operatorname{Bd}\left(U_{1}\right) \cup J \cup B\right) \neq \phi .
$$

Thus if we denote by $N$ the union of all orbit closures which intersect $\operatorname{Bd}\left(U_{1}\right) \cup J \cup B$, we see that $N$ is an invariant continuum which contains no fixed point of $f$ (this follows similarly to the case of $M$ above). Let $V_{1}, \cdots, V_{t}$ be the (finite) number of components of $S^{2}-N$ such that $\operatorname{Fix}(f) \cap V_{j} \neq \phi$ and $V_{j} \subset U_{1}, 1 \leqq j \leqq t$. By Lemma $2, i\left(f, V_{j}\right)=1,1 \leqq j \leqq t$. By Axiom A3,

$$
1=i\left(f, U_{1}\right)=\sum_{j=1}^{t} i\left(f, V_{j}\right)=t
$$

But $J$ separates two points of $\operatorname{Fix}(f) \cap U_{1}$, hence $t>1$. This contradiction shows that Case 1 cannot occur.

Case 2. $\operatorname{Bd}(U)-\operatorname{Bd}\left(U_{1}\right)$ is connected. The proof will be complete if we show that $\operatorname{Bd}(U)-\operatorname{Bd}\left(U_{1}\right)$ is a single point. We assume that $\operatorname{Bd}(U)-\operatorname{Bd}\left(U_{1}\right)$ is a nondegenerate continuum and derive a contradiction.

Assuming $\mathrm{Bd}(U)-\operatorname{Bd}\left(U_{1}\right)$ is a nondegenerate continuum we establish

Claim 1. There is a simply connected invariant domain $C_{v}$ containing two endcuts $A$ and $B$ such that the endpoint of $B$ on $\operatorname{Bd}\left(C_{v}\right)$ is not a fixed point of $f$, and the endpoint of $A$ on $\operatorname{Bd}\left(C_{v}\right)$ is a fixed point of $f$ which is not a limit point of $\operatorname{Bd}\left(C_{v}\right)-\operatorname{Fix}(f)$.

Let $Q$ be a crosscut in $U$ both of whose endpoints lie on

$$
\operatorname{Bd}(U)-\operatorname{Bd}\left(U_{1}\right) \text {. }
$$

Let $V$ be the component of $U-Q$ whose boundary does not intersect $\operatorname{Bd}\left(U_{1}\right),[15,(5.3)$, p. 6]. $\quad V$ is a component of

$$
S^{2}-\left(\left(\operatorname{Bd}\left(U_{1}\right)-\operatorname{Bd}(U)\right) \cup Q\right) \text {. }
$$


Let $p$ be a point of $\mathrm{Bd}(V)-\mathrm{Cl}(Q)$. Note that $p$ is a fixed point of $f$.

Denote by $L$ the union of all orbit closures which intersect $\mathrm{Cl}(Q) . \quad L$ is a continuum. $\quad p$ is not a limit point of $L$ so there is a connected neighborhood 0 of $p$ which misses $L$. Let $A$ be an endcut of $V$ which is contained in 0 . Let $C_{v}$ be the component of

$$
S^{2}-\left(\left(\operatorname{Bd}(U)-\operatorname{Bd}\left(U_{1}\right)\right) \cup L\right)
$$

which contains the endeut $A$. The endpoint of $A$ in $\operatorname{Bd}\left(C_{v}\right)$ has a neighborhood 0 which misses $L$, hence $0 \cap \operatorname{Bd}\left(C_{v}\right) \subset \operatorname{Fix}(f)$.

Let $B^{\prime}$ be an endcut of $V$ with one endpoint $b$ in $C_{v}$ and the other in the crosscut $Q$. Then the component of $B^{\prime} \cap C_{v}$ containing $b$ is the required endeut $B$.

$C_{v}$ is simply connected because $\left(\operatorname{Bd}(U)-\operatorname{Bd}\left(U_{1}\right)\right) \cup L$ is connected, (see [15, (5.3), p. 6] and [10, Theorem 74, p. 217]).

$C_{v}$ is invariant because (a) $\left.(\mathrm{Bd}(U))-\mathrm{Bd}\left(U_{1}\right)\right) \cup L$ is invariant, (b) $\operatorname{Bd}\left(C_{v}\right)$ contains a continuum of fixed points of $f$, and (c) $f$ is orientationpreserving, (for further details see proof of Claim 2 below). The proof of Claim 1 is complete.

Claim 2. The prime end $E$ of $C_{v}$ determined by the endcut $A$ is a fixed prime end of $f$.

Let $S_{1}, S_{2}, \cdots$ be a chain of crosscuts converging to the endpoint $s$ of $A$ and defining the prime end $E$. Since $s$ is not a limit point of $\operatorname{Bd}\left(C_{v}\right)-\operatorname{Fix}(f)$, we may assume that the endpoints of $S_{i}$ are fixed points of $f$ for every $i, i=1,2, \cdots$. We also may assume that every crosscut $S_{i}$ intersects $A$. From the crosscut $S_{i}$ and the endcut $A$ we may construct an open triod $T_{i}$ (see $\S 2$ for definition) whose feet are fixed points of $f$. Since $f$ is orientation-preserving, we see easily that $f\left(T_{i}\right) \cap T_{i} \neq \phi$. (Hence $f\left(C_{v}\right) \cap C_{v} \neq \phi$, and since $\left(\operatorname{Bd}(U)-\operatorname{Bd}\left(U_{1}\right)\right) \cup L$ is invariant, we have $f\left(C_{v}\right)=C_{v}$.)

Since $f\left(T_{i}\right) \cap T_{i} \neq \phi$ for $i=1,2, \cdots$, we see that $\left\{S_{i}\right\}$ and $\left\{f\left(S_{i}\right)\right\}$ are equivalent chains, hence $E$ is a fixed prime end of $f$. The proof of Claim 2 is complete.

Let $T$ be a $C$-transformation of $C_{v}$ onto the open unit disk $D$. Extend the homeomorphism $T f T^{-1}: D \rightarrow D$ to a homeomorphism $h$ of the closed unit disk onto itself. $h$ is orientation-preserving, since $f$ is.

By Claim 2, there is a fixed prime end of $C_{v}$; hence $h$ has a fixed point on $\operatorname{Bd}(D)$. But then, since $h$ is orientation-preserving, every point of $\operatorname{Bd}(D)$ is either a fixed point of $h$ or converges to a fixed point under positive iterates of $h$ [2, Lemma 14].

Consider the endcut $B$ of Claim 1. The endpoint of $B$ on $\operatorname{Bd}\left(C_{v}\right)$ 
is not fixed by $f$, but this endpoint is a principal point of the prime end $F$ determined by $B$. Hence, by Lemma $1, F$ is not a fixed prime end. Hence, if $e$ is the endpoint of $T(B)$ on $\operatorname{Bd}(D), e$ is not a fixed point of $h$. But then, there is a fixed point $m$ of $h$ on $\operatorname{Bd}(D)$ such that $\left\{h^{n}(e)\right\}_{n=0}^{\infty} \rightarrow m$. If $M$ is the prime end of $C_{v}$ corresponding to the point $m$, then by Lemma 1 , every principal point of $M$ is a fixed point of $f$.

Let $X_{1}, X_{2}, \cdots$ be a chain of crosscuts of $C_{v}$ defining the prime end $M$. We claim that for large $j, T\left(X_{j}\right)$ intersects the orbit under $h$ of $T(B)$. To see this we proceed as follows. Let $b$ be the endpoint of $B$ in $C_{v}$. Then the orbit closure of $b$ is contained in $C_{v}$; therefore, the orbit closure of $T(b)$ under $h$ is contained in $D$. In particular, $m$ is not a limit point of the orbit of $T(b)$. Hence, for large $j$, the closure of the crosscut $T\left(X_{j}\right)$ separates $m$ and the orbit of $T(b)$ in $\mathrm{Cl}(D)$. But the other endpoint $e$ of $T(B)$ converges to $m$ under positive iterates of $h$, so for large $j$, there is a positive integer $n$ such that $h^{n}(\mathrm{Cl}(T(B)))$ intersects both components of

$$
\mathrm{Cl}(D)-\mathrm{Cl}\left(T\left(X_{j}\right)\right)
$$

Hence $h^{n}(T(B))$ intersects $T\left(X_{j}\right)$, and our claim is established.

Hence, for large $j, X_{j}$ intersects the orbit under $f$ of $\mathrm{Cl}(B)$.

But the chain $X_{1}, X_{2}, \cdots$ of crosscuts converges to a principal point $q$ of the prime end $M$. But then $q$ is a fixed point of $f$ which is a limit point of the orbit of $\operatorname{Cl}(B)$. Therefore, the union of all orbit closures which intersect $\mathrm{Cl}(B)$ is not a closed set. This contradicts the fact that $f$ is w.a.p.

This final contradiction establishes that $\operatorname{Bd}(U)-\operatorname{Bd}\left(U_{1}\right)$ is a single point. Similarly, $\operatorname{Fix}(f) \cap U_{2}$ is a single point, and so $f$ has exactly two fixed points. The proof of Theorem 3 is complete.

THEOREM 4. Suppose $f: S^{2} \rightarrow S^{2}$ is a w.a.p. orientation-reversing homeomorphism. Then either $f$ has no fixed points, or $f$ is periodic with period 2.

Proof. Suppose $f$ has a fixed point.

Claim. $f$ has more than two fixed points.

Suppose the claim is not true. Let $A$ be an arc intersecting no fixed point, such that $A \cap f(A) \neq \phi$. Denote by $M$ the union of all orbit closures which intersect $A$. $M$ is an invariant continuum containing no fixed points of $f$. Let $U$ be a component of $S^{2}-M$ containing a fixed point of $f$. Then $f(U)=U$ and $U$ is simply connected with a nondegenerate boundary. Let $T$ be a $C$-transformation of $U$ 
onto the open unit disk $D$. Extend $T f T^{-1}$ to a homeomorphism $h$ of the closed unit disk onto itself. $h$ is orientation-reversing, since $f$ is. But then $h$ must have two fixed points on $\operatorname{Bd}(D)$, [16, Theorem 7.3, p. 264]. These fixed points correspond to fixed prime ends of $U$. By Lemma 1, the principal points of these prime ends are fixed points of $f$. This contradicts the assumption that $M$ contains no fixed points of $f$. The proof of our claim is complete.

But now consider the homeomorphism $f^{2}: S^{2} \rightarrow S^{2} . f^{2}$ is orientation-preserving, w.a.p. [6, Theorem 4.24, p. 34 and Theorem 2.33, p. 17], and by our claim, has more than two fixed points. Hence, by Theorem $3, f^{2}=I d$. The proof of Theorem 4 is complete.

Corollary 5. Suppose $f: S^{2} \rightarrow S^{2}$ is a w.a.p. orientationreversing homeomorphism. Then the set of fixed points of $f$ is either empty or is a simple closed curve.

Proof. Follows from Theorem 4 and [3].

7. Orbit closures of weakly almost periodic homeomorphisms on $S^{2}$.

THEOREM 6. Suppose $f: S^{2} \rightarrow S^{2}$ is a w.a.p. orientation-preserving homeomorphism which is not periodic. Then every nondegenerate orbit closure is a 1-dimensional homology 1-sphere.

Proof. $f \neq I d$ so by Theorem 3, $f$ has exactly two fixed points. Let $K$ be a nondegenerate orbit closure. We show that $K$ separates the fixed points of $f$. Suppose not. Then there is a simple closed curve $J$ which separates $K$ and the fixed points of $f$, (connect the fixed points by an arc missing $K$, then "enlarge" the are slightly to obtain a disk whose boundary is $J$ ). We must have $f(J) \cap J \neq \dot{\phi}$, since otherwise every point of $J$ would be a wandering point. Denote by $M$ the union of all orbit closures which intersect $J$. Then $M$ is an invariant continuum which separates $K$ and the fixed points of $f$. Let $U$ be a component of $S^{2}-M$ which intersects $K$. Since every point of $U$ is a nonwandering point, there is an integer $n$ such that $f^{n}(U) \cap U \neq \phi$. Since $M$ is invariant, $f^{n}(U)=U$.

$f^{n}$ is a w.a.p. orientation-preserving homeomorphism [6, p. 34 and p. 17]. $f$ is not periodic, hence $f^{n} \neq I d$, hence by Theorem $3, f^{n}$ has exactly two fixed points. These fixed points are the original fixed points of $f$, and so the domain $U$ contains no fixed points of $f^{n}$. But by Lemma $2, i\left(f^{n}, U\right)=1$. This contradiction shows that the orbit closure $K$ must separate the fixed points of $f$. 
We now show that $K$ is connected. Let $V$ be a component of $S^{2}-K$ containing a fixed point of $f$. Let $B$ be the outer boundary of $V$ with respect to the fixed point of $f$ not in $V$, (see $\S 2$ for definitions). $B$ is connected, [10, Theorem 25, p. 176]. And $V$ and the fixed points of $f$ are invariant, hence $B$ is invariant. But $K$ is a minimal invariant set, and $B \subset K$, hence $B=K$.

$K$ is one dimensional, since outer boundaries contain no interior points.

Finally, $S^{2}-K$ has exactly two components. For, if there were more than two components, then some component $U$ would contain no fixed point of $f$, and we would arrive at the same contradiction as in proving that $K$ separates the fixed points of $f$.

Thus $K$ is a 1-dimensional homology 1-sphere and the proof of Theorem 6 is complete.

REMARK. [12, Example 1] is an example of a w.a.p. orientationpreserving homeomorphism with an orbit closure which is an homology 1-sphere but not a real 1-sphere.

THEOREM 7. Suppose $f: S^{2} \rightarrow S^{2}$ is a w.a.p. orientation-reversing homeomorphism which is not periodic. Then, with two exceptions, every orbit closure is the union of two disjoint homology 1-spheres. The exceptions are (a) a period 2 orbit, and (b) one orbit closure which is an homology 1-sphere (the "axis of reflection").

Proof. $f^{2}$ is a w.a.p., orientation-preserving, nonperiodic homeomorphism. Hence, by Theorems 3 and $6, f^{2}$ has two fixed points, and every nondegenerate orbit closure is an homology 1-sphere. The orbit closure under $f$ of a point $x$ is the union of the orbit closure of $x$ under $f^{2}$ and the orbit closure of $f(x)$ under $f^{2}$. Thus, the two fixed points of $f^{2}$ correspond to a period 2 orbit under $f$, and every other orbit closure under $f$ is the union of two homology 1-spheres which are either disjoint or equal. Let $H$ denote the collection of orbit closures under $f$ which are homology 1-spheres. We show that $H$ has exactly one element.

Let $G$ be the decomposition space whose points are orbit closures under $f^{2}$. Let $w: S^{2} \rightarrow G$ be the natural decomposition map [16, p. 125]. If $K$ is any nondegenerate orbit closure under $f^{2}$, then $w(K)$ is a cut point of $G$, since $K$ separates $S^{2}, w$ is an open map, $[16$, p. 130], and orbit closures are connected. Hence $G$ has exactly two noncut points, (the fixed points of $f^{2}$ ), hence $G$ is an arc, [16, p. 54]. Define a map $g: G \rightarrow G$ by $g(w(K))=w(f(K))$ for all orbit closures $K$ of $f^{2}$. It is easily seen that $g$ is a nontrivial period 2 
map of the arc $G$ onto itself. Fixed points of $G$ correspond to elements of the set $H$ defined above. But $g$ has exactly one fixed point $[16$, p. 264]. The proof of Theorem 7 is complete.

\section{REFERENCES}

1. R. Brown, The Lefschetz Fixed Point Theorem, Scott, Foresman and Co., Glenview, III., 1971.

2. M. Cartwright and J. Littlewood, Some fixed point theorems, Annals of Math., 54 (1951), 1-37.

3. S. Eilenberg, Sur les transformations périodiques de la surface de sphere, Fund. Math., 22 (1934), 28-41.

4. E. Fadell, Recent results in the fixed point theory of continuous maps, Bull. Amer. Math. Soc., 76 (1970), 10-29.

5. W. Gottschalk, Almost periodic points with respect to transformation semi-groups, Annals of Math., 47 (1946), 762-766.

6. W. Gottschalk and G. Hedlund, Topological Dynamics, Amer. Math. Soc. Coll. Pub., Vol. 36, Providence, 1955.

7. E. Hemmingsen, Plane continua admitting nonperiodic autohomeomorphisms with equicontinuous iterates, Math. Scand., 2 (1954), 119-141.

8. B. de Kerékjártó, Über die periodischen transformationen der kreisscheibe und der Kugelfläche, Math. Ann., 80 (1919-1920), 36-38.

9. - Topologische Charakterisierung der linearen abbildungen, Acta Sci. Math. (Szeged), 6 (1934), 235-262.

10. R. L. Moore, Foundations of Point Set Topology, Amer. Math. Soc. Coll. Pub., Vol. 13, Providence, 1962.

11. M. Ohtsuka, Dirichlet Problem, Extremal Length, and Prime Ends, Van Nostrand, New York, 1970.

12. R. Remage, On minimal sets in the plane, Proc. Amer. Math. Soc., 13 (1962), 4147, and "Correction", p. 1000.

13. N. Rutt, Prime ends and indecomposability, Bull. Amer, Math. Soc., 41 (1935), 265-273.

14. E. Spanier, Algebraic Topology, McGraw-Hill, New York, 1966.

15. H. Ursell and L. Young, Remarks on the Theory of Prime Ends, Memoirs of Amer. Math. Soc., No. 3, Providence, 1951.

16. G. Whyburn, Analytic Topology, Amer. Math. Soc., Coll. Pub., Vol. 28, Providence, 1963.

Received June 12, 1972 and in revised form August 24, 1972.

Rutgers, The State University of New Jersey 


\section{PACIFIC JOURNAL OF MATHEMATICS}

\section{EDITORS}

RICHARD ARENS (Managing Editor)

University of California

Los Angeles, California 90024

R. A. Beaumont

University of Washington

Seattle, Washington 98105
J. DUGUNDJI*

Department of Mathematics

University of Southern California

Los Angeles, California 90007

D. Gilbarg and J. Milgram

Stanford University

Stanford, California 94305

\section{ASSOCIATE EDITORS}
E. F. BECKENBACH
B. H. NeumanN
F. WOLF
K. YosHIDA

\section{SUPPORTING INSTITUTIONS}

UNIVERSITY OF BRITISH COLUMBIA
CALIFORNIA INSTITUTE OF TECHNOLOGY
UNIVERSITY OF CALIFORNIA
MONTANA STATE UNIVERSITY
UNIVERSITY OF NEVADA
NEW MEXICO STATE UNIVERSITY
OREGON STATE UNIVERSITY
UNIVERSITY OF OREGON
OSAKA UNIVERSITY

UNIVERSITY OF BRITISH COLUMBIA

UNIVERSITY OF CALIFORNIA

MONTANA STATE UNIVERSITY

UNIVERSITY OF NEVADA

OREGON STATE UNIVERSITY

OSAKA UNIVERSITY
UNIVERSITY OF SOUTHERN CALIFORNIA

STANFORD UNIVERSITY

UNIVERSITY OF TOKYO

UNIVERSITY OF UTAH

WASHINGTON STATE UNIVERSITY

UNIVERSITY OF WASHINGTON

\section{AMERICAN MATHEMATICAL SOCIETY} NAVAL WEAPONS CENTER

* C. R. DePrima California Institute of Technology, Pasadena, CA 91109, will replace J. Dugundji until August 1974. 


\section{Pacific Journal of Mathematics}

\section{Vol. 48, No. $1 \quad$ March, 1973}

Jan Aarts and David John Lutzer, Pseudo-completeness and the product of Baire

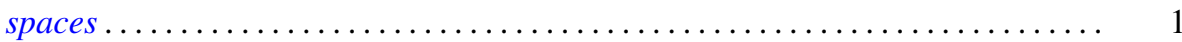

Gordon Owen Berg, Metric characterizations of Euclidean spaces ............ 11

Ajit Kaur Chilana, The space of bounded sequences with the mixed topology ..... . 29

Philip Throop Church and James Timourian, Differentiable open maps of

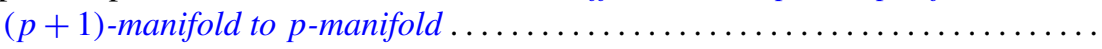

P. D. T. A. Elliott, On additive functions whose limiting distributions possess a finite

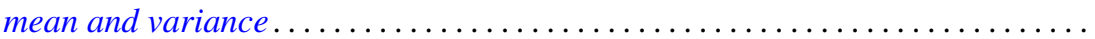

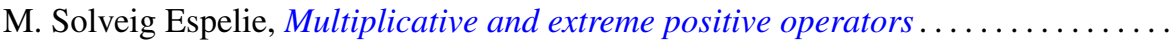

Jacques A. Ferland, Domains of negativity and application to generalized convexity

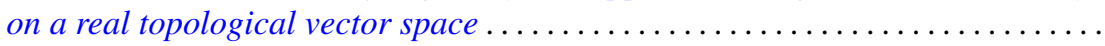

Michael Benton Freeman and Reese Harvey, A compact set that is locally holomorphically convex but not holomorphically convex ...............

Roe William Goodman, Positive-definite distributions and intertwining

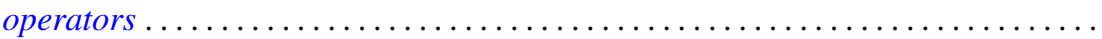

Elliot Charles Gootman, The type of some $C^{*}$ and $W^{*}$-algebras associated with

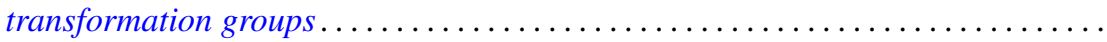

David Charles Haddad, Angular limits of locally finitely valent holomorphic

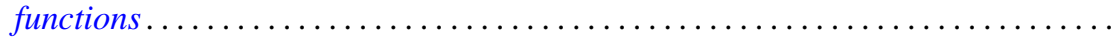

William Buhmann Johnson, On quasi-complements .

William M. Kantor, On 2-transitive collineation groups of finite projective spaces...

Joachim Lambek and Gerhard O. Michler, Completions and classical localizations of right Noetherian rings

Kenneth Lamar Lange, Borel sets of probability measures ......

David Lowell Lovelady, Product integrals for an ordinary differential equation in a Banach space

Jorge Martinez, A hom-functor for lattice-ordered groups .........

W. K. Mason, Weakly almost periodic homeomorphisms of the two sphere ....

Anthony G. Mucci, Limits for martingale-like sequences .......

Eugene Michael Norris, Relationally induced semigroups ...

Arthur E. Olson, A comparison of c-density and $k$-density ......

Donald Steven Passman, On the semisimplicity of group rings of linear groups.

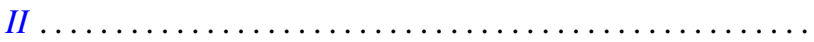

Charles Radin, Ergodicity in von Neumann algebras .

P. Rosenthal, On the singularities of the function generated by the Bergman operator of the second kind.

Arthur Argyle Sagle and J. R. Schumi, Multiplications on homogeneous spaces,

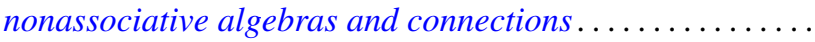

Leo Sario and Cecilia Wang, Existence of Dirichlet finite biharmonic functions on

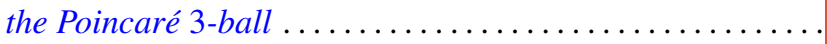

Ramachandran Subramanian, On a generalization of martingales due to Blake ..

Bui An Ton, On strongly nonlinear elliptic variational inequalities.

Seth Warner, A topological characterization of complete, discretely valued

fields. 